\title{
ACCIÓN RACIONAL Y AGENTES COMPLEJOS. Cuestiones de empiria, teoría y metateoría a propósito de los "efectos perversos"
}

\author{
JAVIER L. CRISTIANO
}

Universidad Complutense de Madrid

\section{PALABRAS CLAVE ADICIONALES}

Racionalismo metodológico, Paradigma weberiano, Consecuencias no intencionales, Agregación.

\section{ADDITIONAL KEYWORDS}

Methodological Rationalism, Weberian Paradigm, Unintended Consequences, Aggregation.

RESUMEN. En este artículo se discuten temas teóricos y metodológicos referidos a la investigación de "efectos perversos". En primer lugar, se intenta una delimitación del concepto en tanto tipo particular de "consecuencia no intencional" de la acción social. En segundo lugar, se defiende una interpretación de la postura metodológica de Raymond Boudon, según la cual el estudio de "efectos perversos" ni puede ni debe quedar limitado por los supuestos del "racionalismo metodológico". Tercero, se argumenta la pertinencia de esa afirmación mediante un breve análisis de cuatro casos de "efectos perversos".

SUMMARY. This article discusses some theoretical and methodological issues regarding the research on perverse effects. First, it proposes an analysis of the concept of perverse effect that conceives it as a specific type of unintended consequence of social action. Second, it defends an interpretation of Raymond Boudon's methodological stance, according to which the study of perverse effects cannot and should not be constrained by the assumptions of methodological rationalism. Lastly, the article argues for this interpretation through a brief analysis of four cases of perverse effects.

E-mail: javier_cristiano@hotmail.com

\section{Revista Internacional de Sociología (RIS)}

Tercera Época, nº 30, Septiembre-Diciembre, 2001, pp. 85-104. 
De acuerdo con la conocida interpretación de Stark (1971), la obra de Weber descansa en una metafísica tan sombría como caprichosa, una suerte de mano invisible al revés, según la cual la historia no sólo es ajena a las intenciones humanas, sino que tiene abierta preferencia por lo indeseable. En el escenario de la historia se representa una cruda tragedia, donde las buenas intenciones producen siempre realidades colectivas malévolas. Las famosas páginas finales de La ética protestante y el espiritu del capitalismo, en las que Weber describe el sórdido resultado de la empresa calvinista, son una muestra cabal de la recurrencia que en Weber (dice Stark) adquiere ribetes de obsesión.

Aunque muy discutible, esta interpretación puede admitirse como trasfondo de la obra historiográfica de Weber. No así, en cambio, cuando se trata de sus escritos metodológicos. Pocas ideas más ajenas al espíritu y la lêtra de la epistemología weberiana que la de una voluntad misteriosa que conduce tras bambalinas el destino de la historia, y que lo hace en alguna dirección y con un sesgo específico. Antes bien, una de las enseñanzas primarias y explícitas de su metodología es exactamente la contraria: lo social y lo histórico son fenómenos esencialmente abiertos, que en tanto productos de la acción humana, limitada en saber y poder, dan lugar a configuraciones históricas variables, abiertas y altamente imprevisibles. Investigar las tramas de acción que producen esas configuraciones es la empresa común de las ciencias sociales, incluida la sociología.

En ambos sentidos -el de la historiografía y el de la epistemología- la problemática de los "efectos perversos" tiene sonoros ecos weberianos. Raymond Boudon, quien introdujo el concepto en sociología (Boudon, 1982), reconoce y exalta esta ascendencia. Hablar de "efectos perversos" implica asumir que no existen fuerzas diabólicas (manos invisibles malévolas, o "astucias de la sinrazón") pero que, sin embargo, lo involuntario indeseable está allí como componente irrecusable de la acción y la interacción social real. Reconocer, con el Weber historiador, su desafiante gravitación práctica; y teorizarlo e investigarlo sin prejuicios, como pide el Weber de la epistemología, son sus supuestos de partida.

Pero hay un tercer elemento, importante, que conecta con Weber la problemática de los "efectos perversos". Lo mismo que en los escritos de método de Weber, la discusión contemporánea de los "efectos perversos" mantiene una relación estrecha, compleja y ambivalente con la economía. $\mathrm{Si}$ la "Methodenstreit" alemana del siglo XIX penetró profundamente el esfuerzo fundamentador de Weber, esa suerte de nueva "Methodenstreit" contemporánea acerca de "las fronteras de la economía" ha hecho lo propio con la reflexión y la investigación de los "efectos perversos". De hecho, puede decirse que la cuestión de los "efectos perversos" es un típico problema de modelos "racionalistas" de acción e interacción ${ }^{2}$. 
La intención de este artículo es revisar críticamente esa noción contemporánea de "efectos perversos", con especial (aunque no exclusiva) atención a su dimensión epistemológica. Parto del supuesto de que ese concepto -más precisamente: lo que él encierra de intuición teórica y promesa empíricase encuentra en la intersección de problemas conceptuales, metodológicos y diagnósticos realmente decisivos de la sociología $a_{c t u a l}^{3}$, y que esta ubicuidad lo hace ipso facto digno de atención.

Como el primer inconveniente del concepto es su ambigüedad, mi primer tarea será circunscribir el campo de los "efectos perversos", a fin de fijar un punto de partida común (I). No pretendo que esta sea la única delimitación posible, pero sí que es una aceptable y útil. En segundo lugar, me propongo discutir los fundamentos epistemológicos de su investigación, tal como se encuentran esbozados en los escritos de Boudon (II). Sostendré que esa fundamentación adolece de ambivalencias, pero que puede interpretarse en una dirección que resulta consistente y prometedora. En tercer lugar, intento justificar lo anterior apelando no a argumentos filosóficos, sino a unos pocos casos empíricos (III).

\footnotetext{
'La discusión sobre la "frontera" de la economía se plantea a propósito de la eventual validez del supuesto del actor racional para dar cuenta también de fenómenos tradicionalmente considerados "no económicos". Un texto clásico de defensa de esa postura es el de Becker (1980). Diversos argumentos a favor y en contra se encuentran en los artículos de Coleman, J. y Fararo, T. (1992). En Swedberg (1990) exponen su postura muchos de los más importantes animadores del debate.

${ }^{2}$ Como veremos más adelante (apartado II), Boudon plantea el concepto de "efectos perversos" al amparo de un "racionalismo metodológico" que ha sido interpretado, y utilizado a veces por el propio Boudon, en términos de una generalización de la teoría microeconómica (Parijs, 1981: 300). Por otra parte, muchos casos importantes de "efectos perversos" estudiados y formalizados -como la "contrafinalidad" o la "suboptimalidad"- parten de hecho del supuesto económico del actor intencional-racional.

${ }^{3}$ Muchos de los más importantes diagnósticos de la sociedad occidental contemporánea -por ejemplo, el de la sociedad del riesgo (Beck, 1998), o el del "mundo desbocado" (Giddens, 2000)coinciden en destacar que el distanciamiento de la acción y la intención humana respecto de sus "consecuencias" es una característica dominante (e inquietante) de la sociedad de nuestro tiempo. Pero no se trata solo de una renovación de los diagnósticos modernos sobre la "cosificación" o la "objetivación de la cultura"; estamos ante consecuencias no intencionales graves (el descontrol de los flujos financieros internacionales) e incluso potencialmente desastrosos (el peligro destructivo de la tecnociencia y de sus productos). Estos diagnósticos impulsan un profundo replanteamiento de los problemas clásicos de la "acción social" o la "interacción", pero también de viejas cuestiones metodológicas, tales como la atribución causal de los hechos sociales, la fiabilidad del conocimiento, o el sentido de una intervención crítica del conocimiento sociológico sobre la realidad. Todas estas cuestiones, tanto teóricas como diagnósticas y epistémicas, se encuentran implicadas en un eventual desarrollo de la intuición boudoniana.
} 


\section{EL CAMPO DE LOS "EFECTOS PERVERSOS”}

El concepto de "efectos perversos", tal como es presentado por Boudon, presupone la problemática más conocida de las "consecuencias no intencionales" de la acción social ${ }^{4}$. En realidad, y siendo totalmente fieles a los escritos de Boudon, hay que decir que ambas nociones se superponen en muchos puntos, porque la "perversidad" a la que alude no se refiere tanto a la indeseabilidad de las consecuencias como a su carácter involuntario e imprevisto. Sin embargo, la tradición sociológica ha incorporado el concepto en el sentido que inequívocamente indica la palabra "perverso", esto es, el de consecuencias no intencionales en algún sentido negativas o indeseables ${ }^{5}$. Tenemos que establecer entonces a qué consecuencias puede atribuirse tal carácter.

Podemos recurrir para ello a distintas propuestas de clasificación de las "consecuencias no intencionales". Siguiendo libremente 6 indicaciones de Baert (1991), Ramos (1993) y del propio Boudon (1982: 6), podemos distinguir tres variables primarias: (1) el carácter exitoso o no de la acción respecto de sus intenciones u objetivos; (2) la comparación entre el objetivo o intención que mueve la acción, y el acontecimiento o estado de cosas sometido a análisis (la "consecuencia no intencional"); (3) la valoración, por el actor, del acontecimiento o estado de cosas que resulta imprevistamente de su acción. Según la primera variable el resultado de la acción puede revestir la forma de logro o fracaso. De acuerdo con la segunda, el resultado puede ser simplemente diferente de lo buscado (logrado o no) por el actor, o bien opuesto a ello. En la tercera, el acontecimiento o estado de cosas producido puede ser deseable, indeseable o indiferente. La combinación de opciones da lugar a los siete tipos de consecuencia no intencional de la tabla 1.

Cada agente individual puede alcanzar o no los objetivos que mueven su acción. En el primer caso ("logro de objetivos"), la acción puede producir también otro estado resultante, que puede ser deseable, indeseable o indiferente a ojos del actor. A su vez, cuando el agente fracasa en sus objetivos, puede

\footnotetext{
${ }^{4} \mathrm{La}$ cuestión de las "consecuencias no intencionales" tiene de hecho un estatuto conceptual bastante más consolidado que la de los "efectos perversos". Referencias clásicas al tema -quizás indispensables para comprender las implicancias de los efectos perversos- son Merton (1980), Hayek (1978) y Popper (1957, cap. 14). Desarrollos más recientes pueden encontrarse en Boudon (1990), Merton (1989), Lamo de Espinosa (1990), Ramos (1993) y Elster (1991: cap. 10), entre otros.

"Cfr. por ejemplo la voz "efectos perversos" en el Diccionario de Sociología de Giner, S., Lamo de Espinosa, E., Torres, C., Madrid, Alianza, (1998).

${ }^{6} \mathrm{Me}$ he tomado la libertad de usar estas propuestas en forma parcial y con algunas modificaciones.
} 
Tabla 1.

Consecuencias no intencionales

\begin{tabular}{llll}
\hline Resultado de la acción & $\begin{array}{l}\text { Relación consecuencias } \\
\text { - intenciones de acción }\end{array}$ & $\begin{array}{l}\text { Valoración de las } \\
\text { consecuencias }\end{array}$ \\
\hline Logro de objetivos & Distinto & $\begin{array}{l}\text { Deseable } \\
\text { Indeseable }\end{array}$ & 1 \\
& & Indiferente & 3 \\
Fracaso & Distinto & Deseable & 4 \\
& & $\begin{array}{l}\text { Indeseable } \\
\text { Indiferente }\end{array}$ & 5 \\
& & Indeseable & 7 \\
\hline
\end{tabular}

producir un resultado opuesto al buscado (y por lo tanto lógicamente indeseable para él), o uno simplemente distinto, que a su vez puede resultarle deseable, indeseable $o$ indiferente.

Estas esquemáticas distinciones nos permiten ya algunas precisiones respecto de lo que puede llamarse con propiedad "efectos perversos". Las opciones claramente destacables son la $\mathrm{N}^{\circ} 2$ (el agente logra sus objetivos, pero produce al mismo tiempo, y en forma no prevista, acontecimientos o estados de cosas que para él son indeseables), la $\mathrm{N}^{\circ} 5$ (el agente no sólo fracasa en sus objetivos, sino que produce un resultado adicional que vive como indeseable), y la $\mathrm{N}^{\circ} 7$ (el agente fracasa en sus objetivos, produciendo un resultado que lisa y llanamente se opone a lo que buscaba).

Ahora bien, todo esto parte del supuesto poco realista de un único agente, como si sólo él existiese en el mundo y como si el objeto de su comportamiento (los estados - meta que se propone, y los resultados que efectivamente produce) dependiesen pura y exclusivamente de su actividad. Lo normal es que este no sea el caso, por lo que resulta indispensable extender el modelo a fenómenos de interacción. Supondremos, para simplificar, que existen dos actores -dos actores individuales o dos grupos de actores. Supondremos también que ambos son agentes y no "pacientes", en el sentido de que contribuyen, sea en proporción igual o distinta, a producir el resultado o consecuencia que es objeto de análisis (el "efecto perverso"). Mantenemos, desde luego, el supuesto de la no intencionalidad de las consecuencias. Bajo tales supuestos, es probable que, concluido el proceso de interacción, los actores hayan obtenido resultados distintos (en términos de logro o fracaso) y/o que valoren en forma diferente las consecuencias resultantes. Las combinaciones lógicas posibles suman 
RIS

RETISTA INTERNACIONAL DE SOCIOLOCÍA

No 30, Septiembre-Diciembre, 2001

JAVIER L. CRISTIANO

28 casos, de los que retenemos, dada su relevancia para nuestros fines, las 13 siguientes:

$2,2-5,5-7,7-1,5-1,7-2,5-2,6-2,7-3,5-3,7-4,5-4,7-5,7$

Todos los casos tiene en común dos características: hay un efecto de la interacción que no estaba en los planes de ningún actor -y en tal sentido es "involuntario" o "no intencional"-, y al menos uno de los dos actores o grupos tiene motivos de insatisfacción respecto del resultado agregado de las acciones de todos. Ahora bien, esos rasgos comunes se presentan a su vez en formas cualitativamente diversas según sean las razones y los modos de la insatisfacción. En la tabla 2 se muestran los matices.

Existe un primer conjunto de casos -que he llamado "malestar universal"en los que ambos actores o conjuntos de actores resultan insatisfechos, sea por razones formalmente idénticas $(5,5 ; 2,2 ; 7,7)$ sea por razones divergentes $(2,5$; 2,6; 2,7 y 5,7). En el segundo conjunto -"contradicciones"- se agrupan los casos en que la actitud frente al resultado de la interacción es opuesta. Así por ejemplo, en el caso extremo de [1,5], lo que para uno de los actores representa una acción exitosa a la que se añade una consecuencia adicional deseable, supone para el otro un fracaso al que suma la indeseabilidad de las consecuencias ${ }^{7 *}$.

Desde luego, la tabla no tiene más intención que mostrar un panorama de los "efectos perversos". Sin embargo, sugiere ya algunas interesantes direcciones de investigación. En primer lugar, la existencia de efectos perversos no supone -y no tiene por qué suponer- que todos los actores involucrados comparten la misma insatisfacción proveniente de la misma "situación-problema". Es decir, no todos están igual de mal por la misma razón. Esta afirmación sería francamente perogrullesca si muchos de los "efectos perversos" mejor conocidos y formalizados no supusiesen justamente lo contrario, a saber: malestar universal por una misma y única razón. Así, por ejemplo, el conocido "dilema del prisionero" -verdadero paradigma de interacción perversa- nos describe la insatisfacción unánime de un conjunto de actores orientados al mismo fin. Lo mismo ocurre con el fenómeno de la contrafinalidad (Elster, 1994: cap. 5), con los de "masa crítica" (Schelling, 1989: 83 y ss.) o con los "modelos

\footnotetext{
7 "Contradicción" puede ser un nombre controvertido debido a la historia y a las implicaciones de ese concepto. Mi uso es aquí amplio y bastante sencillo. De todas formas, pensar algunos usos clásicos de "contradicción" como "efecto perverso" (o a la inversa, pensar los efectos perversos "contradictorios" a luz de los análisis tradicionales de "contradicción") es estimulante proyecto del que contamos ya con aportes relevantes (cfr. Elster, 1994, caps. 4 y 5).
} 
Tabla 2.

Efectos pervesos

\begin{tabular}{llll}
\hline Tipo & Caso & \multicolumn{1}{c}{ Actor A } & \multicolumn{1}{c}{ Actor B } \\
\hline Malestar & 5,5 & Fracaso + consecuencia indeseable & Fracaso + consecuencia indeseable \\
universal & 2,2 & Logro + consecuencia indeseable & $\begin{array}{l}\text { Logro + consecuencia indeseable } \\
\end{array}$ \\
& 7,7 & Fracaso + consecuencia opuesta & Fracaso + consecuencia opuesta \\
& 2,5 & Logro + consecuencia indeseable & Fracaso + consecuencia indeseable \\
& 2,6 & Logro + consecuencia indeseable & Fracaso + consecuencia indiferente \\
& 2,7 & Logro + consecuencia indeseable & Fracaso + consecuencia opuesta \\
& 5,7 & Fracaso + consecuencia indeseable & Fracaso + consecuencia opuesta \\
Contradic- & 1,7 & Logro + consecuencia deseable & Fracaso + consecuencias opuestas \\
ciones & 1,5 & Logro + consecuencia deseable & Fracaso + consecuencias indeseables \\
& 3,5 & Logro + consecuencias indiferentes & Fracaso + consecuencia indeseable \\
& 3,7 & Logro + consecuencias indiferentes & Fracaso + consecuencias opuestas \\
& 4,5 & Fracaso + consecuencias deseables & Fracaso + consecuencias indeseables \\
& 4,7 & Fracaso + consecuencias deseables & Fracaso + consecuencias opuestas \\
\hline
\end{tabular}

de umbral" de acción colectiva (Granovetter, 1991). En todos estos casos tenemos formalizaciones de "efectos perversos" que no reconocen matices en la insatisfacción de los actores. La tabla 2 muestra hasta qué punto deberían considerarse un tipo muy específico de "efecto perverso", y sugiere el interés de elaborar modelos que contemplen también al resto de los casos.

En segundo lugar, la inclusión de "efectos perversos" con "ganadores y perdedores" ("contradicciones") permite recoger una realidad empírica que, no obstante ser obvia, tiene importante significación práctica y epistemológica. En lo práctico se vincula con las motivaciones de cambio de cada actor, y por lo tanto con la cuestión de la evitabilidad, evitación y reversibilidad de los "efectos perversos". Actores desigualmente satisfechos o insatisfechos tendrán posiblemente desigual disposición para modificar el curso de su acción. En lo epistemológico, la diferencia plantea de un modo concreto, aunque poco habitual, el problema del estatuto y el uso del conocimiento sociológico. Así, si en el caso de la contrafinalidad -donde todos pierden por igual- es de esperar que el conocimiento sea una contribución directa a superar el problema, no es obvio que tal ocurra cuando el conocimiento ilumina mejor a cada actor la naturaleza de su interés, y sobre todo su incompatibilidad respecto del de sus pares. En la sección III vuelvo sobre esta cuestión.

Estas rápidas pinceladas son sugerentes, creo, sobre el potencial de una investigación amplia de los "efectos perversos". De todos modos, el paso preliminar de esa empresa es discutir los supuestos en que apoyarla, y a ello se dedica la mayor parte de lo que sigue. 


\section{FUNDAMENTOS: RACIONALISMO METODOLÓGICO Y AGENCIA COMPLEJA}

Las distinciones de la sección anterior se basan en una visión muy simplificada de la acción humana, del intercambio material y simbólico (interacción) y de sus consecuencias. Suponen entre otras cosas que los actores se orientan siempre por "objetivos" o "fines", que las consecuencias de sus actos son unívocas y claras, y que la percepción y evaluación de esas consecuencias no reviste inconveniente alguno, ni para el actor ni para el investigador. Consideré lícito usar estas simplificaciones para dar precisión al concepto, pero es evidente que la investigación y teorización propiamente dicha son más exigentes. Voy a explorar con Boudon -y en cierta medida también contra Boudon- la respuesta a la pregunta por los "fundamentos" de la investigación de "efectos perversos".

Para Boudon, el estudio de los "efectos perversos", como el de la sociología en general, se inscribe en la "tradición individualista" de las ciencias sociales y asume el "paradigma weberiano" de explicación (Boudon, 1986). Ese paradigma puede resumirse en una fórmula,

$$
\mathrm{M}=\mathrm{M}\left\{m_{i}\left[\mathrm{~S}\left(\mathrm{M}^{\prime}\right)\right]\right\}
$$

tal que: siendo $\mathrm{M}$ un suceso a explicar (el efecto perverso), ha de considerarse función de un conjunto de acciones individuales $m_{i}$; estas acciones, a su vez, deben considerarse como "dependientes" de una situación (S), que depende a su vez de un dato o variable $M$ ', de nivel macrosocial, o por lo menos del mismo nivel en el que tiene lugar el explanandum M. En otras palabras: remitimos el acontecimiento o estado de cosas a acciones humanas; las acciones a su situación; y la situación a acontecimientos o estados de cosas antecedentes que, a través de las acciones, se vinculan con el explanandum.

Ahora bien, este paradigma es sólo una estructura lógica, que puede "rellenarse" con contenidos muy diversos. La discusión de fondo es cuál de esos contenidos es más apropiado, y en particular, qué sentido debemos atribuir a la palabra "depende" cuando se afirma que "las acciones individuales $\left(\mathrm{m}_{\mathrm{i}}\right)$ son dependientes de una situación (S)". La respuesta que privilegia Boudon es ampliamente conocida: adhesión a un "racionalismo metodológico" (Boudon, 1981; Parijs, 1981), y más específicamente, a un tipo de racionalidad que denomina "compleja", y que diferencia de los modelos standard de racionalidad. Merece la pena clarificar este punto, porque de él se derivan consecuencias sustantivas respecto del significado de la investigación de "efectos perversos".

Que la racionalidad sea "compleja" significa para Boudon que no puede establecerse en abstracto, sino sólo en referencia al contexto en que se desarrolla. A diferencia de los modelos de racionalidad que toman como parámetro un 
conocimiento "científico" externo (vgr.: el concepto parsoniano de "acción racional") el parámetro de la racionalidad boudoniana es la especificidad del contexto, y la información que sobre él ha acumulado el propio actor. Desde esta perspectiva, es incorrecto calificar de "irracional" a, por ejemplo, las aspiraciones de estudiantes de bajos recursos, que el investigador observa como injustificadamente limitadas y que atribuye, supongamos, a una "irracional" fidelidad a su cultura clase (Boudon, 1986: 50). Si tuviésemos suficiente información sobre el contexto -es decir, si efectivamente comprendiésemos el sentido de esa acción- hallaríamos su propia racionalidad, que no está fundada en conocimientos expertos y valores ajenos al medio (como los del mundo científico académico), sino en el contexto mismo y en su estructura particular.

Es los trabajos de Boudon pueden encontrarse muchas ilustraciones de este tipo, algunas ciertamente sorprendentes por su radicalidad ${ }^{8}$. Sin embargo, basta este ejemplo para mostrar la ambigüedad de la "racionalidad compleja", e incluso la dudosa pertinencia de denominarla de tal modo, "racionalidad". Todo lo que nos quiere decir Boudon es que los actores tienden a obrar con una cierta razonabilidad, en el sentido de que en cierto modo "saben lo que hacen" y obran de un modo que es "comprensible" a la luz de sus creencias y su saber respecto de la situación. Y que si un comportamiento se nos presenta a primera vista como carente de sentido, impulsivo o "ciego", haremos bien en suponer que somos nosotros, y no el actor, quien carece de la información suficiente.

Van Parijs (1981: 302) advierte también esta ambigüedad, pero considera a Boudon como un "racionalista metodológico", umbral 3 de su continum de opciones ("individualismo", "intencionalismo", "racionalismo" y "utilitarismo" metodológicos ${ }^{9}$ ). En esta interpretación, la postura de Boudon queda hermanada con la de los defensores abiertos del "racionalismo" strictu sensu y/o del "utilitarismo", como Elster, Coleman, Gary Becker o el propio van Parijs ${ }^{10}$. Una hermandad que el mismo Boudon no rechaza, y que avala en algunos de sus más importantes trabajos empíricos, en los que utiliza por ejemplo el modelo de la Rational Choice Theory ${ }^{11}$.

\footnotetext{
${ }^{8}$ Por ejemplo, afirma que incluso el comportamiento guiado por la tradición es "racional", puesto que cuando un actor "acepta" la tradición es porque le atribuye sentido y efectividad adaptativa (Cfr. Boudon, 1986: 51).

${ }^{9}$ Estas cuatro opciones (individualismo, intencionalismo, racionalismo, utilitarismo) se implican entre sí de derecha a izquierda, pero no a la inversa. Así, es lógicamente posible aceptar el individualismo metodológico rechazando el resto de las opciones, mientras que aceptar el "utilitarismo" implica aceptar también el "racionalismo", el "intencionalismo" y el "individualismo".

${ }^{10}$ Cfr. Elster (1989); Coleman (1990); Becker (1980); Parijs (1981).

"Véanse por ejemplo los estudios empíricos de Boudon (1982).
} 
RIS

REVISTA INTERNACIONAL DE SOCIOLOGIA

№30, Septiembre-Diciembre, 2001

JAVIER L. CRISTIANO

Sin embargo, es dificil encontrar en Boudon alguna afirmación epistemológica explícita que haga inevitable esta interpretación. Y más aún, no hay nada que impida leer su insistencia en la "racionalidad compleja" en un sentido mucho más próximo a otras orientaciones de la sociología contemporánea, igualmente respetuosas de la contextualidad de la acción, pero carentes de compromiso alguno con la idea de "racionalidad". Me refiero, por ejemplo, a las visiones de la agencia humana que podemos encontrar en autores como Giddens o Bourdieu, en las que el axioma de que "los hombres saben lo que hacen" -es decir, que no son "drogados culturales" o "idiotas que juzgan"- se traduce en una estructura conceptual diversificada, sutil y flexible, que permite una captación efectivamente "compleja" de la acción humana ligada al contexto.

Lo que se juega en esta interpretación no es una cuestión de preferencias teóricas, o un simple detalle semántico (a qué debemos o no llamar "racionalidad"). Lo que está en juego son los supuestos de la investigación y de la teoría, y más específicamente: los supuestos respecto del qué y el para qué del conocimiento sociológico -en nuestro caso, el conocimiento de los "efectos perversos". Las visiones que van Parijs llama "racionalistas" y "utilitaristas" no sólo comparten el sustrato antropológico del homo economicus (o por lo menos, un "modelo de portafolio"12 del actor social) sino también un modelo de conocimiento basado (1) en el ideal de transparencia y precisión, cuya expresión paradigmática es la formalización matemática; (2) la orientación hacia la predicción, ligada a la eficacia práctica del comportamiento. Son estos ideales los que justifican la simplificación de la acción humana al esquema propositivo medios - fines, y la asunción (más o menos violenta, según los casos) de que "los hombres son racionales hasta tanto se demuestre lo contrario"13. Asumir visiones efectivamente "complejas" de la acción y la interacción, como las que proponen Giddens o Bourdieu, implica ante todo poner en duda la validez universal de estos supuestos, aceptando que no toda investigación y no toda teoría están obligadas a compartirlos, y que no resultan indiscutibles para todos los "objetos". No supone negar su valor, pero sí (y sobre todo) rechazar su universalidad.

\footnotetext{
${ }^{12}$ La expresión "modelo de portafolio" fue introducida por Hindess (1988) para referirse a las teorías de la acción en las que se presupone que los actores portan de un contexto a otro (como en un "portafolio") un conjunto de deseos y creencias que operan en todas sus decisiones.

${ }^{13}$ Los partidarios de la elección racional coinciden en afirmar la conveniencia de presuponer que el comportamiento es "racional" aún allí donde la evidencia empírica parece indicar lo contrario, invirtiendo (digamos) la carga de la prueba: lo que hay que probar no es la racionalidad, sino la irracionalidad, es decir, la completa imposibilidad de ajustar el comportamiento a parámetros de ajuste medios - fines. Los artículos clásicos de Becker (1980) y Freedman (1986) exponen $\mathrm{y}$ argumentan esta postura.
} 
Este desplazamiento, del "racionalismo metodológico" a la agencia efectivamente compleja, es una lectura válida, y creo que una mejor lectura, de la insistencia de Boudon en la "complejidad" del obrar humano y en la contextualidad de su "sentido". Pero además, es compatible con otras dos insistencias importantes, ampliamente asumibles también, de sus escritos metodológicos. Primero, que la sociología es ante todo una ciencia de lo singular, preocupada por la explicación de lo concreto y, por lo tanto, con vocación más histórica que sistemática. Todo el esfuerzo de la sociología se orienta a dar cuenta de los cómo y los por qué de acontecimientos o estados de cosas particulares, partiendo de una sana desconfianza respecto de las generalizaciones y esquemas concluyentes. $Y$ en segundo lugar, su insistencia en un individualismo metodológico amplio, cuya vocación no es la búsqueda estéril del detalle (reduccionismo), sino más bien impedir los errores de las generalizaciones macro transportadas de un contexto a otro. La esencia del paradigma weberiano es precisamente esta: remitir el explanandum siempre a acciones, y nunca directamente a "macroestados". Tanto uno como otro postulado parecen más próximos a una visión de la agencia humana que no prejuzgue respecto de sus fundamentos, y que se abra a la observación lisa y llana de sus mecanismos y formas específicas.

Esta lectura de la epistemología boudoniana podría defenderse como plasmación del "paradigma weberiano" en general, esto es, como forma válida de la explicación sociológica en general. Sin embargo, mi interés es defenderla sobre todo cuando se trata de "efectos perversos", en virtud de dos características que distinguen a éstos de otros objetos de investigación y teorización.

(1) En la medida en que los "efectos perversos" son acontecimientos o estados de cosas que los propios partícipes viven como indeseables, existe en ellos una mayor preocupación por la intervención práctica correctiva que pueda orientar el conocimiento. La invocación de una teoría realmente compleja de la acción humana, unida a la sensibilidad respecto de lo singular e idiosincrásico de cada caso, contribuyen a desactivar los peligros de una sociología excesivamente abstracta y sistemática, mal predispuesta por definición a esos matices, y propensa a violentar la especificidad cuándo se convierte en recomendación política concreta. Estos riesgos están a la vista en el tipo de práctica política que con mucha frecuencia alienta y respalda la economía, ciencia ejemplar para la mayoría de los autores filiados en la empresa "racionalista" y "utilitarista".

(2) La indeseabilidad de los "efectos perversos", sumada a su carácter de agregación "ciega", involuntaria, tornan notoriamente importante la cuestión de la responsabilidad de su producción. El individualismo metodológico -la pretensión de referir los "efectos perversos" a concretas acciones individuales- contribuye a la tarea práctica de la elucidación de responsabilidad, e indirectamente por tanto, a la evitación de los "efectos perversos". 


\section{RIS}

Estas justificaciones incluyen fuertes consideraciones de valor, y podrían ser desarrolladas y discutidas en esa dirección, la de lo ético normativo. Mi estrategia en el apartado siguiente será menos pretenciosa: trataré de mostrar cómo podría operar el conocimiento, y distintos "tipos" de conocimiento, en casos concretos y bastante conocidos de "efectos perversos".

\section{PROCESOS Y SABERES}

Las cuestiones epistemológicas y metodológicas suelen discutirse a cierta distancia -casi siempre muy grande- de las realidades empíricas. Y aún cuando en las mismas discusiones se subraye que son problemas inevitablemente unidos a marcos socio-históricos particulares, se suelen desatender los muchos matices y detalles del mundo empírico, que a veces desafian y condicionan más de lo que se piensa los argumentos filosóficos globales. Por eso quisiera cerrar este artículo invocando unos pocos casos empíricos de "efectos perversos", para dar verosimilitud a las afirmaciones del apartado anterior. Creo que es una estrategia argumental adecuada, y tiene la ventaja de reconocer un postulado prácticamente unánime de la teoría social y la epistemología contemporánea: el de la inevitable implicación del discurso sociológico en su. objeto. Esta implicación, llámese "carácter crítico", "efecto de teoría" o "reflexividad"14, es un tema crucial en los "efectos perversos", debido a la tensa relación que se da en ellos entre la no intencionalidad del fenómeno y las posibles consecuencias de su conocimiento. La pertinencia de producir un tipo $\mathrm{u}$ otro de conocimiento, basado en uno u otro género de presupuestos, es también (aunque no sólo) cuestión de realidad empírica.

Ciclo de la telaraña. Un caso muy conocido y estudiado de "efecto perverso" es el descubierto y discutido por los economistas a propósito de un problema de los productores de cerdos californianos: el yerro sistemático de sus predicciones sobre el futuro del mercado. Los criadores de cerdos tienen que decidir cada año el volumen de su producción para el año siguiente, tomando como parámetro los precios del año en curso. Cuando, movidos por la cotización baja, deciden producir pocos cerdos y apostar a otros productos, el precio del cerdo se eleva y pierden la oportunidad de un excelente negocio. A la inversa, cuando movidos por los buenos precios del

\footnotetext{
13 Sobre el carácter critico de la sociología, cfr. Giddens (1987: cap. 4); sobre el "efecto de teoría", Bourdieu (1996: 133); para una aproximación a las distintas versiones de la "reflexividad", la primera parte de García Selgas (1999).
} 
año actual hacen una apuesta fuerte para el próximo, los precios se deterioran y las pérdidas son cuantiosas. La investigación muestra que el problema reside en la generalización de las expectativas: es la propia decisión de cada productor individual, amplificada, lo que produce mayor oferta y con ello baja de precios; y a la inversa, es la escasa producción lo que genera un desequilibrio con la demanda que eleva los precios.

Aquí tenemos un típico éxito del modelo de conocimiento que preconiza la economía y, más ampliamente, los modelos "racionalistas" y "utilitaristas". La economía se enfrenta al enigma de los productores con sus herramientas habituales: la reducción ideal típica de la acción humana a parámetros de racionalidad, la agregación matemática de las consecuencias de acción, las funciones de utilidad, la noción de equilibrio de mercado, etc. No sólo logra de este modo resolver el enigma (mostrar con nitidez y precisión la dinámica del problema), sino también indicar un curso de acción óptimo. Los productores de cerdos saben ahora que no hay más "causa" de su problema que sus propias decisiones cuando ellas se generalizan. El conocimiento claro y unívoco es en este caso una condición necesaria (aunque no suficiente) de la solución del problema. De hecho, podría intervenir una nueva variable (por ejemplo, cambios en el gusto de los consumidores) que tornen ocasionalmente acertados los pronósticos, pero la solución deliberada del problema presupone su conocimiento.

Acción colectiva: dilema del prisionero. Los trabajadores de una empresa desean aumento salarial y deciden ir a la huelga. Es mejor para todos que todos la acaten, porque de este modo la presión será mayor, y mayor también la probabilidad de éxito. Sin embargo, cada trabajador individual tiene la tentación de no unirse a la huelga (evitando así represalias o descuentos en su salario), porque de todos modos disfrutará del aumento de sueldo si sus compañeros lo logran. Esta acción (no cooperar) es la más racional individualmente, pero conduce a la irracionalidad cuando se generaliza. El "efecto perverso" se produce precisamente en este caso: todos pensaron lo mismo, nadie acudió a la huelga, y el beneficio posible no se ha alcanzado.

Como se sabe, el dilema del prisionero ha sido ampliamente estudiado en la teoría matemática de juegos, una de las ramas más fértiles de la Rational Choice Theory. Quizás la más intensa y estimulante de las discusiones ha sido la de su "solución", donde descuellan estudios brillantes como los clásicos de Olson (1992) y Axelrod (1986). Estos análisis son sin embargo esencialmente formales, y un plano bastante relegado de la discusión es el de su impacto práctico: ¿qué efecto tiene el conocimiento del problema sobre su solución?. La respuesta no es univoca, y depende de factores que el dilema, en su estructura formal, no contempla. Una opción es que, frente a un concreto fracaso de la acción colectiva, el conocimiento formalizado cumpla su objetivo de hacer manifiesto lo latente. 
En tal caso, el modelo formaliza como acción estrictamente racional, ejecutada por maximizadores de preferencias constantes y ordenadas, lo que en la realidad es una intuición semiconsciente pero efectivamente operante. El conocimiento contribuye en este caso a desatar el nudo de un fracaso colectivo, y las recomendaciones de "incentivos selectivos" o "reiteración del juego" funcionan como orientación política eficaz. Ahora bien, el conocimiento del dilema puede derivar también en un tipo especial de "profecía de autocumplimiento", si es que el conocimiento viene a reafirmar y/o iluminar la racionalidad de la no-cooperación. En tal caso, la racionalidad deja de ser un expediente metodológico -suponer actores racionales a fin de reducir complejidad y hacer posible la explicación-y pasa a ser un modelo de acción que uno o varios actores pueden de hecho asumir. Esto significa que el conocimiento del dilema puede crear el dilema allí donde no existía.

La cuestión está lejos de ser trivial, y algunas situaciones estrictamente económicas (como los pánicos bursátiles) sugieren hasta qué punto puede afectar el estatuto de la propia teoría ${ }^{15}$. Lo único que quiero sugerir aquí, sin embargo, es hasta qué punto la visión simplificada de la acción humana puede tener consecuencias que trascienden el campo teórico para deslizarse en la esfera práctica. Enfrentar un fracaso concreto de acción colectiva con el instrumento conceptual del Dilema del Prisionero, implica procesar de un modo muy especial fenómenos como (por ejemplo) la adhesión psicológica a una causa sindical, o el sentimiento de pertenencia y fidelidad a una tradición política. Estos fenómenos pueden estar presentes, y su gravitación puede ir mucho más allá de lo que permite reconocer su reducción al esquema medios - fines. Pero lo que se resiente en tal caso no es el esquema formal de la teoría -que permanece incólume- sino la realidad empírica sobre la que se proyectan "soluciones".

Espiral de racismo. En los años de entreguerras, los sindicatos norteamericanos establecieron impedimentos para la afiliación de trabajadores de raza negra. $\mathrm{Su}$ argumento fue la supuesta escasa lealtad sindical de los negros, propensos a no acatar las huelgas. Según el análisis de Merton (1965: 421), el proceso se desenvuelve en un círculo vicioso. Puesto que los sindicatos controlan buena parte del mercado laboral, la marginalidad de los negros respecto de la estructura sindical les impide conseguir empleos con facilidad. Ello los coloca en tal situación de desprotección, que la oferta de trabajar en días de huelga (proveniente de empleadores en apuros) es muy dificil de desechar. El hecho de que muchos trabajadores negros obren de esa manera "confirma" su deslealtad a ojos de los sindicalistas, que afianzan y radicalizan sus medidas de exclusión, reiniciando el proceso y dándole nuevo impulso.

\footnotetext{
${ }^{15}$ Véase al respecto el muy interesante e instructivo trabajo de Izquierdo (1996).
} 
Nos enfrentamos aquí a un "efecto perverso" bastante distinto de los anteriores. Los componentes del proceso están organizados circularmente, en una espiral de agravamiento que, ligada a su propia lógica, aumentaría indefinidamente tanto la solidez del prejuicio como su consecuencia, a saber: la marginación de los trabajadores negros. La estrategia conceptual de Merton es sencilla: dilucida el proceso como un caso de "profecía de autocumplimiento", y atribuye a los actores una suerte de racionalidad de sentido común, que hace intuitivamente plausible su explicación. De hecho, todos damos por sentado que, estando en lugar de los trabajadores negros, no dudaríamos en emplearnos durante las huelgas; que muy posiblemente contrataríamos trabajadores negros en tales casos si fuésemos empresarios en apuros; e incluso que, si creyésemos sinceramente que los negros son desleales, veríamos en su comportamiento una confirmación de nuestra creencia. También en este caso, por tanto, tenemos una simplificación bastante notoria de las "razones" que los participantes tienen para actuar, y un retrato de la acción humana ciertamente indiferente al bagaje conceptual de la propia sociología. Ahora bien, ¿por qué es necesario apelar a ese bagaje, si conseguimos sin él una explicación sencilla y suficiente del problema?. Una vez más, la respuesta depende de condiciones empíricas. En la explicación de Merton, el nudo del problema es el prejuicio de los sindicalistas; son ellos quienes tiene la llave del problema, y quienes deben "comprender" la lógica del proceso y su responsabilidad en él. Pero la explicación considera aproblemática nada menos que la cuestión de la formación, el refuerzo y la modificación del prejuicio. En particular, da por sentada la sinceridad de la declaración pública de los sindicalistas ("los trabajadores negros son sindicalmente desleales"), sin sospechar -como sugeriría la más simple lectura psicológica del caso- que se trata de la racionalización pública de un malestar más profundo, más arraigado, y desde luego mucho menos racional. En otras palabras, se supone que el prejuicio de los sindicalistas no es más que un error, producto de una observación errónea o incompleta de la "realidad". Esto puede ser así, pero también puede no serlo, y sólo munidos de un instrumento de análisis más o menos sofisticado respecto de (en este caso) el comportamiento prejuicioso, seremos capaces de saberlo.

$\mathrm{Al}$ igual que en los casos anteriores, lo que está en juego es la hipotética relación entre el conocimiento generado y el desenvolvimiento posterior de la realidad investigada. Si diésemos por cierta la explicación de Merton, bien podría suceder que el reconocimiento del mecanismo por parte de los sindicalistas no derive -como "razonablemente debiera suceder"- en una revisión de las medidas de exclusión, sino en nuevas y más sofisticadas justificaciones de esa exclusión. En tal caso, la razón profunda del prejuicio no habría sido revelada, y la intervención práctica resultaría cuanto menos inocua. 


\section{RIS}

Autoperpetuación de la desigualdad de oportunidades. Paul Willis (1977) ha investigado el modo en que la cultura de las clases populares traba una relación conflictiva con la cultura "oficial", tal como ella se manifiesta en las prácticas y las normas del mundo escolar. En particular, mostró que ciertos comportamientos rebeldes de los alumnos de clases obreras de Birmingham son realizados a partir de un conocimiento preconciente, pero complejo y meticuloso, de la estructura de autoridad escolar y de sus fisuras. El "procesamiento" de esa rebeldía por el sistema de normas y sanciones de la escuela tiende a expulsarlos prematuramente del sistema formal de educación, ingresando en inferioridad de condiciones al mercado de trabajo y reproduciendo asi la subordinación de clase. Esa reproducción de subordinación resulta así una consecuencia no intencionada de la acción de los alumnos y de los miembros de la comunidad escolar.

Nuestro cuarto y último caso representa el polo opuesto de las formalizaciones del tipo de la Elección Racional o la teoría de juegos, de donde partimos. Paul Willis no sólo toma en serio las conceptualizaciones más detalladas de la agencia humana, sino que confia menos en ellas que en su propia y desprejuiciada observación de cientos de pequeños detalles y de lo que ellos indican o sugieren al investigador atento. Es así como descubre, por ejemplo, el significado de los silencios o las bromas cotidianas como indicadores de un minucioso conocimiento práctico, no verbalizado y difícilmente verbalizable, de la institución en la que se producen. En tal sentido, Willis lleva a la práctica en forma indiscutiblemente consecuente la exigencia boudoniana de suponer una "racionalidad compleja" ligada a la situación. Solo que no se limita a dispensar una relación de medios y fines, y acepta sumergirse en el complejo mundo intersubjetivo que tiene lugar todos los días en las aulas y los pasillos de la escuela. Logra reconstruir de esta manera una "racionalidad", que es en realidad una sofisticada relación comprensible entre condiciones, vida subjetiva y comportamiento.

El tipo de repercusión práctica que puede imputarse a esta investigación es ciertamente distinto de los casos anteriores. En primer lugar, es bastante difícil que una indagación tan detallada pueda traducirse en implicancias normativas precisas y unívocas. Antes bien, la investigación parecería impulsar más a una actitud que a unos comportamientos concretos. Los maestros podrían advertir por ejemplo las consecuencias remotas (no intencionales) del ejercicio de su autoridad, pero de ello sería difícil inferir modos de actuar concretos e indiscutibles capaces de evitarlos. Los jóvenes alcanzarían de hecho una autocomprensión muy diferente de sus propias prácticas, pero tampoco para ellos se trataría de un simple "obrar en consecuencia", dado que lo que está en juego es un mundo de vida frente al que es problemática por definición la intervención racional deliberada. En segundo lugar, se trata de una investigación tan particularizada, que resulta evidente la imposibilidad de generalizar sus 
argumentos. Lo que Willis afirma que sucede, sucede en un colegio de un barrio obrero de Birmingham, y todo lo que puede hacer en referencia a casos parecidos es sugerir algunas hipótesis.

En suma, la investigación parece desafectar cualquier decisión categórica y general que la invoque como apoyo. $\mathrm{Y}$ al contrario, parece invitar a una reflexividad deliberada del conocimiento por los propios agentes, los únicos capaces de atribuirle un sentido orientado a cursos de acción correctivos.

Recapitulemos brevemente. En el apartado anterior se defendió una interpretación de la metodología de Boudon, según la cual: (1) el postulado de la "racionalidad compleja" no tiene por qué ser interpretado exclusivamente en el sentido del "racionalismo metodológico", y es plenamente compatible con la asunción de modelos de agencia efectivamente "complejos", ajenos a la idea de "racionalidad"; (2) el individualismo metodológico es una estrategia especialmente apropiada al tratarse de "efectos perversos", debido a su relación práctica con la imputación y la autoimputación de responsabilidad; (3) la investigación de "efectos perversos" tiene, como la de la sociología en general, una vocación más histórica que sistemática.

Los casos muestran (aunque en trazos gruesos) el funcionamiento de estos principios. En todos ellos tiene lugar una adhesión al individualismo metodológico, pero en una gradiente que va desde la racionalidad estricta del homo economicus (el ciclo de la telaraña y el dilema del prisionero) hasta un caso de "descripción densa" de la agencia humana y la interacción institucional (la autoperpetuación de las desigualdades), pasando por el caso intermedio de la espiral de racismo. Los ejemplos señalan el vínculo que une la simplificación en la descripción de la acción humana, la generalidad del conocimiento (su capacidad para desprenderse del caso concreto) y la precisión y claridad de las recomendaciones prácticas que alienta. Cuanto mayor es la simplificación descriptiva de la acción, más general parece hacerse el conocimiento, y más precisa y clara la recomendación práctica que se desprende de él. Y a la inversa, cuando más "densa" -y por lo tanto menos simplificada- es la descripción de la acción, el conocimiento parece perder generalidad y las recomendaciones prácticas tornarse más abiertas y ambiguas.

No se trata de una relación inalterable, sino de una afinidad: de lo simple con lo general y lo preciso; y de lo "denso" con lo particular y con lo ambivalente. Lo que los ejemplos ponen de manifiesto es que la claridad y la precisión de las recomendaciones prácticas no tiene por qué ser deseable. Que puede ser peligrosa, y que es más peligrosa cuando más probable sea que en la "simplificación" se pierdan detalles importantes, es decir, "detalles" que no son tales. Y que el hecho de que esta condición se de o no -que existan detalles que no son tales- depende del caso empírico, y esta es la virtud de discutir la cuestión al amparo de realidades particulares, históricas. 
RIS

REUTSTA INTERNaCIONAL DE SOCIOLOGIA

N`30. Septiembre-Diciembre. 2001

JAVIER L. CRISTIANO

La conclusión es sencilla, pero no irrelevante: cualquier afirmación epistemológica universal referida a la acción -del tipo "adhesión (incondicional) al racionalismo metodológico"- queda desafectada no sólo por la diversidad y variedad de los fenómenos empíricos de "efectos perversos", sino sobre todo por la diversidad y variedad de las relaciones que el conocimiento traba con la realidad empírica de los efectos perversos. Lo poco y breve que hemos dicho sobre esta relación no hace más -ni pretende más- que ilustrar esos matices y reclamar su investigación detallada, pero alcanza para mostrar hasta qué punto los "efectos perversos" no son sólo "trampas" que la sociología viene a desactivar, sino trampas en las que puede caer y a las que incluso puede ayudar a tender.

\section{CONCLUSIONES}

En su Presidential Address ante la American Sociological Asociation, en 1992, James Coleman llama a la sociología a volcar todos sus esfuerzos hacia la "reconstrucción racional de la sociedad" (Coleman, 1993). Curiosamente, utiliza la misma expresión ("reconstrucción racional de la sociedad") usada por Max Horkheimer para argumentar a favor de su "teoría crítica". Difícilmente puedan imaginarse, como es obvio, posiciones filosóficas y temperamentos teóricos más irreconciliables. Todo lo que quiere Coleman es que la sociología ayude a construir instituciones sociales, en un tiempo en que -afirma- las sociedades han dejado de sustentarse en lazos normativos y tradicionales, y marchan rápidamente hacia la completa artificialidad y voluntariedad de sus formas organizativas. La sociología que alienta y desarrolla Coleman es un caso extremo de lo que Horkheimer condenaba en términos de "racionalidad instrumental"; una ciencia que no tiene nada que decir sobre la racionalidad de los "fines", y que aspira a decirlo casi todo sobre la racionalidad de los "medios".

La investigación de "efectos perversos", tal y como ha sido esbozada en este artículo, llama la atención al mismo tiempo sobre la tentación y sobre el riesgo de propuestas como la de Coleman. Por un lado, los "efectos perversos" reclaman a la sociología en su dimensión de conocimiento experto, capaz de proporcionar los medios adecuados para fines que a ella misma -a la sociologíano le competen. Por su propia definición, los "efectos perversos" suponen la vivencia de un malestar, y la demanda consiguiente de "solución", "acción correctiva" y proposición "política". La tentación consiste entonces en pensar la sociología como una caja de herramientas ya dispuestas para su uso, según el modelo de la microeconomía que el propio Coleman impulsa y que orienta en importante medida (como hemos visto) la investigación de "efectos perversos". Pero al mismo tiempo, los "efectos perversos" muestran hasta qué punto las "herramientas" de la sociología no son tales, sino más bien variables de los 
mismos fenómenos que se propone "corregir". Su involucramiento en los "efectos perversos" no es instrumental y externo, sino activo y local. Lo que he tratado de mostrar es que ignorar esta realidad puede ser menos un defecto epistemológico que una falta de responsabilidad.

\section{BIBLIOGRAFÍA}

AXELROD, R. (1986), La evolución de la cooperación, Madrid, Alianza.

BAERT, P. (1991), “Unintended consequences: a typology and examples", International Sociology, $\mathrm{n}^{\circ} 6,2$.

BECK, U. (1998), La sociedad del riesgo. Hacia una nueva modernidad, Barcelona, Paidós.

BECKER, G. (1980), "El enfoque económico del comportamiento humano", Información comercial española, 557, enero.

BOUDON, R. (1981), La lógica de lo social, Madrid, Rialp.

(1982), The Unintented Consequences of Social Action, Macmillan Press Ltd., London.

(1986), "Individual Action, Aggregation Effects and Social Change”, Theories of Social Change, Oxford, Polity Press.

(1990), "The two Facets of the Unintended Consequences Paradigm", en Clark, J.C. S. Modgil, S. Modgil, Robert Merton. Consensus and Controversy, London, Falmer Press.

BOURDIEU, P. (1996), Cosas dichas, Barcelona, Gedisa.

COLEMAN, J. (1990), Foundations of Social Theory, Cambridge, Belknap Press of Harvard University Press.

(1993), "The Rational Reconstruction of Society - 1992 Presidential Address", American Sociological Review, vol. 28, February (1-15).

COLEMAN, J. y T. FARARO [eds.] (1992), Rational Choice Theory. Advocacy and Critique, California, Sage Publications.

ELSTER, J. (1989), Ulises y las sirenas, México, FCE.

(1991), Tuercas y tornillos, Barcelona, Gedisa.

(1994), Lógica y sociedad, Barcelona, Gedisa.

FREEDMAN, M. (1986), "El método de la economia positiva", en Hann, F. y M. Holis, Filosofia y teoria económica, México, FCE. 
RIS

GARCÍA SELGAS, F. (1999). "La reflexividad y el supuesto-sujeto", en García Selgas, F. y R. Ramos Torre, Globalización, riesgo, reflexividad: tres temas de la teoria social contemporánea, Madrid, CIS.

GIDDENS, A. (1987), Las nuevas reglas del método sociológico, Buenos Aires, Amorrortu.

(2000), Un mundo desbocado, Madrid, Taurus.

GRANOVETTER, M. (1991), "Modelos de umbral de conducta colectiva", en F. Aguiar (comp.), Intereses individuales y acción colectiva, Madrid, Pablo Inglesias.

HAYEK, F. (1978). "The Results of Human Action but not of Human Design", New studies in Philosophy, Politics, Economics and the History of Ideas, Londres, Routledge and Kegan Paul.

HINDESS, B. (1988), Choice, Rationality, and Social Theory, Boston, Unwin Hyman. *

IZQUIERDO MARÍN, J. (1996). "Equilibrio económico y racionalidad maquínica”, Política y sociedad, 21, pp. 89-111, Madrid.

LAMO DE ESPINOSA, E. (1990), La sociedad reflexiva, Madrid, CIS.

OLSON, M. (1992), La lógica de la acción colectiva, México, Limusa - Noriega.

MERTON, R. (1980), “Las consecuencias imprevistas de la acción social”, Ambivalencia sociológica y otros ensayos, Madrid, Espasa-Calpe.

(1989), "Unanticipated Consequences and Kindred Sociological Ideas: A Personal Gloss", en Mongardini, C. y S. Taboni, L'opera di R. K. Merton e la sociologia contemporanea, EGIG (Edizioni Culturali Internazionali Genova).

(1965), Teoria y estructura sociales, México, FCE.

PARIJS, P. (1981), "Sociology as general economics", Archives Européenes de Sociologie, 22, pp. 299-324.

POPPER, K.(1957), La sociedad abierta y sus enemigos, Buenos Aires, Paidós.

RAMOS TORRE, R. (1993), "Una aproximación a las paradojas de la acción social”, en Lamo de Espinosa, E. y J. Rodriguez Ibáñez (ed.), Problemas de teoria social contemporánea, CIS, Madrid.

SCHELLING, T. (1989), Micromotivos y macroconducta, México, FCE.

STARK, W. (1971), "Max Weber y la heterogonía de los fines", en Parson, T. (comp.), Presencia de Max Weber, Buenos Aires, Nueva Visión.

SWEDBERG, R. (1990), Economics and Sociology. Redefining their boundaries: conversations with economists and sociologists, Princeton, Princeton University Press.

WILLIS, P. (1977), Learning to Labour, Farnborough, Saxon House. 